\title{
Semiclassical gravitational effects around global monopole in Brans-Dicke theory
}

\author{
${ }^{*}$ F.Rahaman and P.Ghosh
}

\begin{abstract}
In recent past, W.A.Hiscock [ Class.Quan.Grav. (1990) 7,6235 ] studied the semi classical gravitational effects around global monopole. He obtained the vacuum expectation value of the stress-energy tensor of an arbitrary collection of conformal mass less free quantum fields ( scalar, spinor and vectors ) in the space time of a global monopole. With this stress-energy tensor, we study the semi classical gravitational effects of a global monopole in the context of Brans-Dicke theory of gravity.
\end{abstract}

Topological defects could be produced at a phase transition in the early Universe. The study of topological defects has wide applicability in many areas of physics. In the cosmological arena, defects have been put forward as a possible mechanism for structure formation. Monopole is one of the topological defects, which arises when the vacuum manifold contains surfaces, which can't be shrunk continuously to a point [1]. These monopoles have Goldstone fields with their energy density decreasing with distance as $\frac{1}{r^{2}}$. They are also found to have interesting features in the sense that a monopole exerts practically no gravitational force on its surrounding non relativistic matter but the space time around it has a deficit solid angle [1]. At first, Barriola and Vilenkin (BV) [2] showed the existence of such a monopole solution resulting from the breaking of global $S 0(3)$ symmetry of a triplet scalar field in a Schwarzschild background.

In 1990, Hiscock [3] obtained the vacuum expectation value of the stress energy tensor of an arbitrary collection of conformal mass less free quantum fields (scalar, spinor and vectors) in the space-time of a static monopole. Taking these non zero vacuum expectation value of the stress energy tensors as a source, he solved the semi classical Einstein equations for the quantum perturbations ( to the first order in $\hbar$ ) of the metric.

${ }^{0}$ Pacs Nos : $98.90 \mathrm{cq}, 04.20 \mathrm{Jb}, 04.50$

Key words and phrases : Semi classical approach, Brans-Dicke theory, Global monopole.

${ }^{*}$ Dept. of Mathematics, Jadavpur University, Kolkata-700 032, India

E-Mail:farook_rahaman@yahoo.com 
The Brans-Dicke theory of gravity is widely studied and well known alternative theory of Einstein gravity. This theory properly accommodates both Mach's Principle and Dirac's large number hypothesis. In this theory a scalar field $\phi$ is coupled to gravity with coupling constant $\omega[4]$.

It is widely accepted that gravity is not given by the Einstein's action at the sufficient high energy scales. The present unified theories indicates that a gravitational scalar field should exist besides the metric of the spacetime. It is believed that in the early stages of the Universe, the coupling to matter of the scalar field would be the same order as that of the metric. Since topological defects are formed during phase transition, so it is not unnatural to study them in a scalar tensor theory of gravitation as that of Brans-Dicke theory.

In recent, Several authors have extended the monopole solution of BV to scalar tensor theory [5], Lyra geometry [6], Dilaton gravity [7], Einstein Kalb-Ramond field [8], C-field theory [9], Tachyonic field [10], Einstein-Cartan theory [11], etc .

In this letter, we would like to discuss semi classical gravitational effects around global monopole in Brans-Dicke theory. We have taken the same vacuum expectation value of the stress energy tensor as obtained by Hiscock and set equal to the Brans-Dicke field equations to solve for the quantum perturbations (to the first order in $\hbar$ ) of the metric. According to BV, the spacetime of global monopole may be written as

$$
d s^{2}=d t^{2}-d r^{2}-r^{2} \alpha^{2} d \Omega_{2}^{2}
$$

with, $\alpha^{2}=1-8 \pi G \eta^{2}$, where $\eta$ is the scale of symmetry breaking.

According to classical theory, the trace of the energy stress tensor vanishes if the Lagrangian is conformally invariant. Whereas corresponding quantized theory it acquires a trace during renormalization. This trace is a geometrical scalar which contains derivatives of the metric tensor $[3,12]$. The trace of the vacuum stress energy for a conformally coupled massless free field is given by the anomaly constructed from the curvature tensors of the spacetime as

$$
T_{a}^{a}=\frac{1}{2880 \pi^{2}}\left[a C_{i j k l} C^{i j k l}+b\left(R_{i j} R^{i j}-\frac{1}{3} R^{2}\right)+c \square R+d R^{2}\right]
$$

The constants a, b, c and d depends on the conformal scalar field under consideration and other symbols have their usual meaning as in Riemannian geometry.

The Brans-Dicke field equations are taken as

$$
\begin{gathered}
R_{a b}=\frac{8 \pi}{\phi}\left[T_{a b}-\frac{1}{2} g_{a b} \frac{(2 \omega+2)}{(2 \omega+3)} T\right]+\frac{\omega}{\phi^{2}} \phi_{, a} \phi_{, b}+\frac{\phi_{; a ; b}}{\phi} \\
\nabla^{\alpha} \nabla_{\alpha} \phi=\frac{1}{2 \omega+3} 8 \pi T
\end{gathered}
$$

where $\phi$ is the scalar field, $\omega$ is a dimension less coupling constant and $T$ denotes the trace of $T_{a}^{b}$, the energy momentum tensors of the matter fields. 
In our consideration, the vacuum expectation values of the stress tensors of the quantum fields can be set in to the Brans-Dicke equations (3)and(4) in the semi classical approach to the quantum theory of gravity:

$$
\begin{gathered}
R_{a b}=\frac{8 \pi}{\phi}\left[<T_{a b}>-\frac{1}{2} g_{a b} \frac{(2 \omega+2)}{(2 \omega+3)}<T>\right]+\frac{\omega}{\phi^{2}} \phi_{, a} \phi_{, b}+\frac{\phi_{; a ; b}}{\phi} \\
\nabla^{\alpha} \nabla_{\alpha} \phi=\frac{1}{2 \omega+3} 8 \pi<T>
\end{gathered}
$$

Here $\langle T\rangle$ denotes the trace of $\left\langle T_{a b}\right\rangle$, the vacuum stress energy momentum tensors. Here the geometrical units are used with $G=c=1$ and $\hbar \approx 2.612 \times 10^{-66} \mathrm{~cm}^{2}$.

At first, Hiscock has studied the quantum effects due to monopole background in the matter fields. Using trace anomaly given in equation (2), one can find the vacuum expectation value of the stress energy tensor of a conformally coupled massless scalar field of the global monopole metric given in (1) as [3]

$$
<T_{a}^{b}>=\frac{\hbar}{r^{4}}[-D,-(C+D),(C+D),(C+D)]
$$

where ,

$C=\frac{1}{1440 \pi^{2}}\left[n_{s c}+3 n_{s p}+12 n_{v d}-12 n_{v \zeta} \frac{2 \alpha^{2}+1}{3 \alpha^{2}-1}\right]\left[\frac{\left(1-\alpha^{2}\right)\left(3 \alpha^{2}-1\right)}{\alpha^{4}}\right]$

with $n_{s c}, n_{s p}, n_{v d}$ and $n_{v \zeta}$ are respectively, the number of scalar two components spinor, dimensional regularized vector zeta function regularized vector fields present and D is dimensionless constant, like $\mathrm{C}$, depends on the number and spin of the component fields present and on the metric parameter $\alpha$ ( given in eq.(1)).

Now we are trying to find the first order in $\hbar$ corrections to the BV monopole in Brans Dicke theory treating the effects of the vacuum stress energy as small perturbations. The first order in $\hbar$ correction to the metric may be determined by solving the linearized Brans Dicke equations. We assume the correction metric of the form as

$g_{r r}=1+f(r), g_{t t}=1+g(r)$

We also assume the correction of the Brans Dicke scalar field as

$\phi=\phi_{0}+\epsilon(r)$

where $\phi_{0}$ is a constant which may be identified with $\frac{1}{G}$ when $\omega \rightarrow \infty$ ( $\mathrm{G}$ being the Newtonian gravitational constant ). 
Here the functions $f, g$ and $\epsilon$ should be computed to the first order in $\hbar$. In this approximation it is easy to see that [5]

$$
\frac{\phi^{\prime}}{\phi}=\frac{\epsilon^{\prime}}{\phi_{0}}, \frac{\phi^{\prime \prime}}{\phi}=\frac{\epsilon^{\prime \prime}}{\phi_{0}}
$$

with $f, g, \epsilon \ll 1$.

With these approximations, field equations (5) and (6) yield

$$
\begin{gathered}
\frac{1}{2} g^{\prime \prime}+\frac{g^{\prime}}{r}=\frac{8 \pi \hbar}{\phi(2 \omega+3) r^{4}}[\omega(C+2 D)+(2 C+3 D)] \\
-\frac{1}{2} g^{\prime \prime}+\frac{f^{\prime}}{r}=-\frac{8 \pi \hbar}{\phi(2 \omega+3) r^{4}}[\omega(3 C+2 D)+(4 C+3 D)]+\frac{\epsilon^{\prime \prime}}{\phi_{0}} \\
f+\frac{1}{2}\left(f^{\prime}-g^{\prime}\right) r=\frac{8 \pi \hbar}{\phi(2 \omega+3) r^{2}}[\omega(C+2 D)+(2 C+3 D)]+r \frac{\epsilon^{\prime}}{\phi_{0}} \\
\epsilon^{\prime \prime}+2 \frac{\epsilon^{\prime}}{r}=\frac{8 \pi \hbar C}{(2 \omega+3) r^{4}}
\end{gathered}
$$

Now solving the above equations, we get the following solutions:

$$
\begin{gathered}
g=\frac{8 \pi \hbar}{\phi_{0}(2 \omega+3) r^{2}}[\omega(C+2 D)+(C+3 D)]-\frac{k_{1}}{r} \\
f=-\frac{8 \pi \hbar}{\phi_{0}(2 \omega+3) r^{2}}[2 D \omega+(C+3 D)]-\frac{\left(k_{1}+\frac{2 k}{\phi_{0}}\right)}{r} \\
\epsilon=\frac{4 \pi \hbar C}{(2 \omega+3) r^{2}}-\frac{k}{r}
\end{gathered}
$$

( where $k, k_{1}$ are integration constants ).

Both the above constants $k, k_{1}$ will be taken as zero, as it is impossible to dimensionally construct a value of the constants $\mathrm{k}$ and $k_{1}$ which are linear in $\hbar$.

It is currently known that solutions of Brans-Dicke field equations do not always go over general relativity solutions when $\omega \rightarrow \infty$. However, in our semi classical approach, we see that in the limit $\omega \rightarrow \infty$, our solution reduces to Hiscock's solution, which is given by

$$
d s^{2}=\left[1+4 \pi \hbar \frac{(C+2 D)}{r^{2}}\right] d t^{2}-\left[1-\frac{8 \pi \hbar D}{r^{2}}\right] d r^{2}-r^{2} \alpha^{2} d \Omega_{2}^{2}
$$




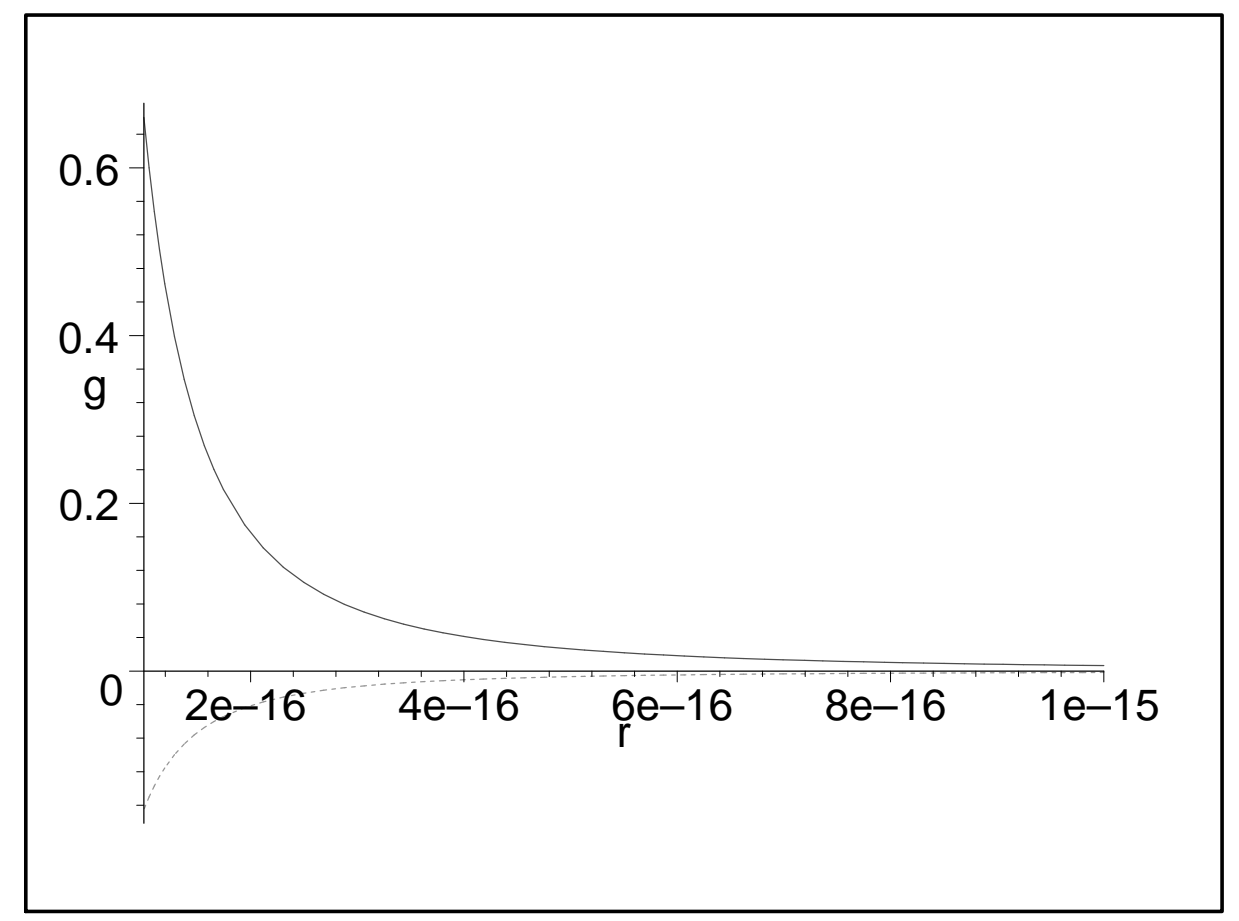

Figure 1: We show the variation of $\mathrm{g}$ w.r.t. $\mathrm{r}$ for different values of $\omega$ (here we assume, $\hbar=1.05459 \times 10^{-34} \mathrm{~J}$-sec, $\left.\phi_{0} \sim \frac{1}{G}, C=1, D=2\right)$. The solid curve for $\omega=500$ [13] and dotted curve for $\omega=-1.42$. 


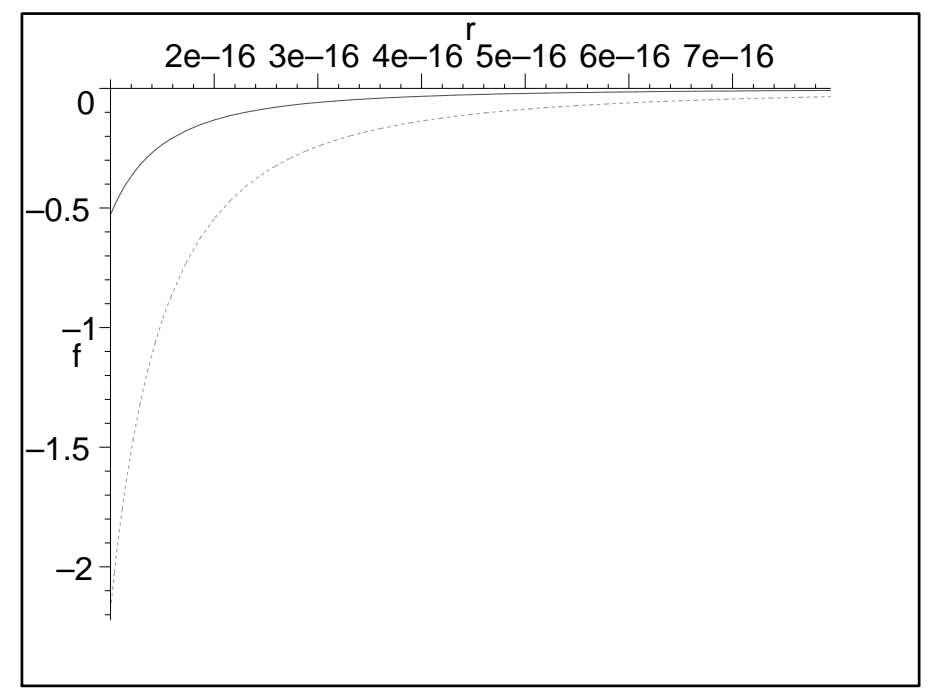

Figure 2: We show the variation of $\mathrm{f}$ w.r.t. $\mathrm{r}$ for different values of $\omega$ (here we assume, $\hbar=1.05459 \times 10^{-34} \mathrm{~J}$-sec, $\phi_{0} \sim \frac{1}{G}, C=1, D=2$ ). The solid curve for $\omega=500$ and dotted curve for $\omega=-1.42$.

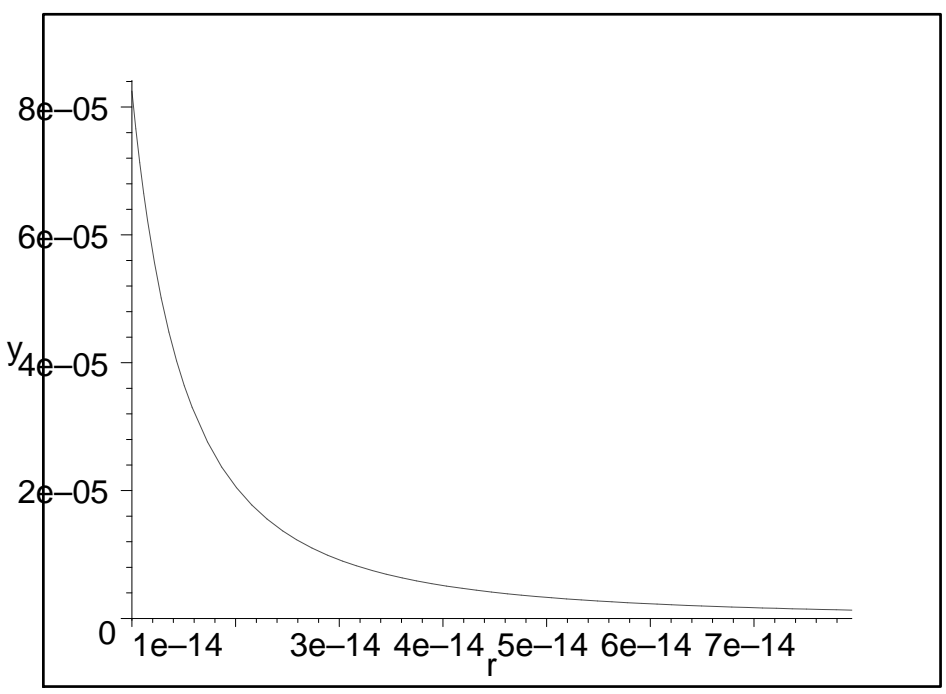

Figure 3: We show the variation of $y \equiv \epsilon$ w.r.t. $\mathrm{r}$. Here we assume, $\hbar=1.05459 \times 10^{-34}$ J-sec, $\phi_{0} \sim \frac{1}{G}, C=1, D=2$ and $\omega=-1.42$. 


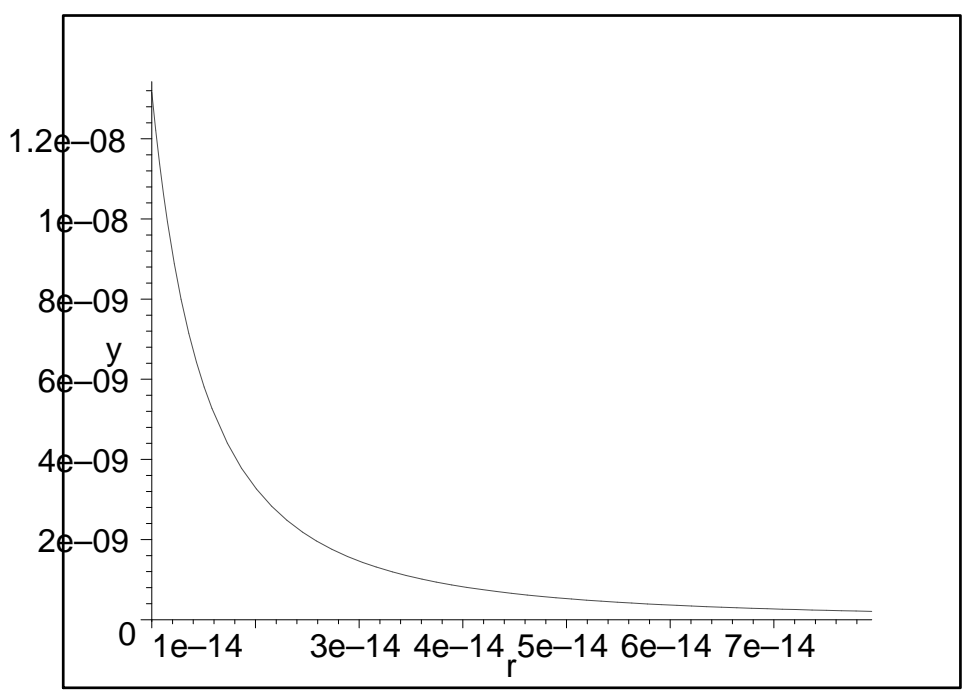

Figure 4: We show the variation of $y \equiv \epsilon$ w.r.t. $\mathrm{r}$. Here we assume, $\hbar=1.05459 \times 10^{-34}$ J-sec, $\phi_{0} \sim \frac{1}{G}, C=1, D=2$ and $\omega=500$.

We shall now discuss the gravitational force of the monopole on the surrounding matter. The radial component of the acceleration $\left(A^{r}\right)$ acting on a test particle in the gravitational field of the monopole is given by

$$
A^{r}=V_{; 0}^{r} V^{0}
$$

For a co-moving particle $V^{a}=\frac{1}{\sqrt{900}} \delta_{0}^{a}$.

Hence using the line element (7) and solutions (19) and (20), one can calculate $A^{r}$, which becomes

$$
A^{r}=\frac{-\frac{16 \pi \hbar H}{\phi_{0}(2 \omega+3) r^{3}}}{\left[1+\frac{8 \pi \hbar H}{\phi_{0}(2 \omega+3) r^{2}}\right]^{2}}
$$

where, $H=[\omega(C+2 D)+(C+3 D)]$.

Here one can see that the gravitational force varies with the radial distance. One can note that $A^{r}$ may take negative or positive values depending on $\omega$. When, either $\omega>$ $\operatorname{Max}\left(-\frac{C+3 D}{C+2 D},-\frac{3}{2}\right)$ or $\omega<\operatorname{Min}\left(-\frac{C+3 D}{C+2 D},-\frac{3}{2}\right)$, then $A^{r}<0$ and this indicates that particle accelerates towards the monopole in the radial direction in order to keep it rest. This implies that monopole has a repulsive influence on the test particle [14]. If, either $-\frac{C+3 D}{C+2 D}<\omega<-\frac{3}{2}$ or $-\frac{3}{2}<\omega<-\frac{C+3 D}{C+2 D}$, then $A^{r}>0$ and this indicates that particle accelerates away from the monopole in the radial direction in order to keep it rest. This implies that monopole has an attractive influence on the test particle [14]. 


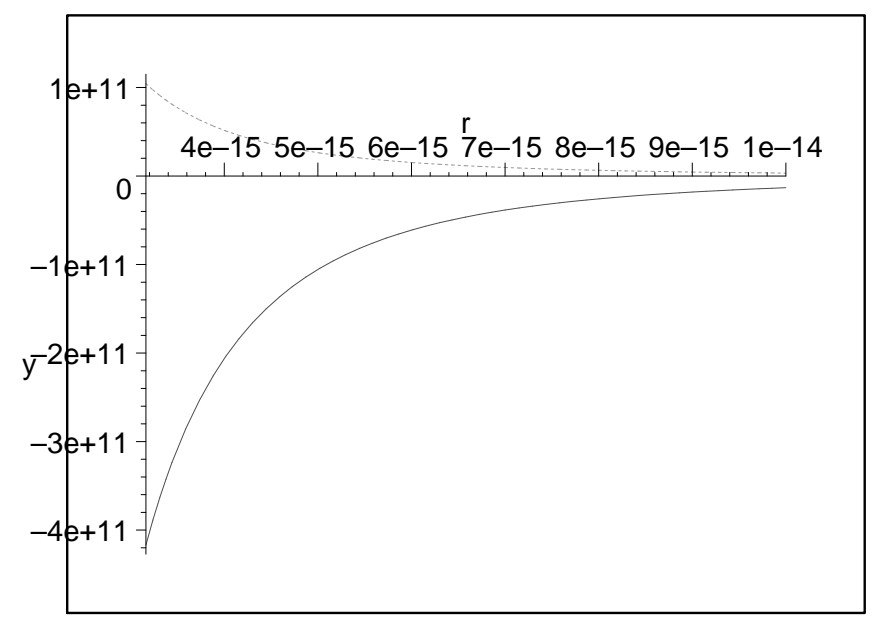

Figure 5: We show the variation of $y \equiv A^{r}$ w.r.t. $\mathrm{r}$ for fixed $\omega$. Here we assume, $\hbar=1.05459 \times 10^{-34} \mathrm{~J}$-sec, $\phi_{0} \sim \frac{1}{G}, C=1, D=2$. The solid curve for $\omega=500$ and dotted curve for $\omega=-1.42$.

It is some interest to calculate the deficit solid angle in the above field.

The deficit solid angle $\delta$ may be defined as

$$
\delta(r)=4 \pi\left[1-\frac{g_{\Omega \Omega}(r)}{r^{2}}\right]
$$

in any spherical symmetric spacetime, where $R(r)$ is the proper radius, $R=\int \sqrt{g_{r r}} d r$. For the first order correction to the metric (1),

$$
R=r+4 \pi \hbar \frac{(2 \omega D+C+3 D)}{\phi_{0}(2 \omega+3) r}
$$

Thus in this case, we obtain,

$$
\delta(r)=4 \pi\left[1-\alpha^{2}+8 \pi \hbar \frac{(2 \omega D+C+3 D) \alpha^{2}}{\phi_{0}(2 \omega+3) r^{2}}\right]
$$

Here one can note that the deficit solid angle is a function of $\mathrm{r}$ and the first order correction to the deficit angle is gradually decreasing with the increase of the radial distance. One can also note that for a fixed radial distance, the deficit solid angle decreases with the increase of $\omega>0$ and it is increasing with decreasing $\omega<-\frac{3}{2}$. It deserves to mention that as $\omega \rightarrow \infty$, this deficit solid angle will take the same value as obtained by Hiscock in general relativity case.

In summary, we have studied the semi classical gravitational effects of a global monopole in Brans-Dicke theory. We have assumed the same vacuum expectation values of the stress energy tensors as obtained by Hiscock and see that when $\omega \rightarrow \infty$, Hiscock's solution is recovered. We see that our monopole exerts repulsive gravitational force as well as attractive gravitational force on its surrounding non relativistic matter depending on the Brans-Dicke coupling constant $\omega$. This effect is absent in general relativity case [15]. Analogously to the general relativity case the curved space-time of the monopole presents a deficit solid angle. 


\section{Acknowledgements:}

F.R is thankful to DST, Government of India for providing financial support. We are also grateful to the anonymous referee for his valuable comments and constructive suggestions.

\section{References}

[1] Kibble T.W.B., J. Phys. A 9, 1387(1976); A.Vilenkin and E.P.S. Shellard, (1994) Cosmic String and other Topological Defects (Camb. Univ. Press)

[2] M. Barriola and Vilenkin. A, Phys. Rev. Lett. 63, 341(1989)

[3] W.A.Hiscock, Class.Quan.Grav. 7, 6235 ( 1990 )

[4] Brans. C and Dicke. R.H, Phys.Rev. 124, 925(1961)

[5] Barros. A and Romero. C, Phys.Rev.D 56, 6688 (1997); A.Banerjee et al, Class.Quant.Grav. 15, 645(1998); T.Lee and B.Lee, Phys.Rev.D. 69, 127502(2004);

F.Rahaman et al, Mod.Phys.Lett.A 22, 971 (2007)

[6] F Rahaman, Int.J.Mod.Phys.D 9, 775 (2000)

[7] O.Dando and R. Gregory, gr-qc/9709029

[8] F Rahaman et al, Int.J.Mod.Phys.D 12, 1837 (2003)

[9] F. Rahaman et al, Astrophys.Space Sci. 302, 171 (2006)

[10] X.Li and D.Liu, Int.J.Mod.Phys.A 20, 1627(2005);

M.Kalam et al, arXiv: 0707.0053 [gr-qc] ( to appear in Gen.Rel.Grav. )

[11] F. Rahaman et al, Astrophys.Space Sci. 299, 167 (2005)

[12] W.A.Hiscock , Phys.Lett.B 188, 317 ( 1987 ); Rahaman F et al, Czech.J.Phys. 54, 785 (2004)

[13] C M Will, Theory and Experiments in Gravitational Physics (1993), Cambridge University Press

[14] A. A. Sen, Phys.Rev. D 60, 067501 (1999)

[15] S.Chakraborty and F.Rahaman, Int.J.Mod.Phys.D 9, 155 (2000) 